\title{
Erratum
}

\section{Erratum to: Comparison Between Total Knee Arthroplasty and MCIC (autologous bone marrow mesenchymal-cell-induced-chondrogenesis) for the Treatment of Osteoarthritis of the Knee}

Jang Mook Kim² $\cdot$ Ju Rang $\mathrm{Han}^{3} \cdot$ Asode Ananthram Shetty ${ }^{4}$ Seok Jung Kim

Nam Yong Choi ${ }^{1} \cdot$ Jun Soo Park ${ }^{1}$

${ }^{1}$ Department of Orthopaedic Surgery, College of Medicine, The Catholic University of Korea

${ }^{2}$ Graduate School of Healthcare Management and Policy, The Catholic Institute for Health Management Research,The Catholic University of Korea

${ }^{3}$ Department of Nursing, Seojeong University of Korea

${ }^{4}$ Canterbury Christ Church University, Faculty of Health and Social Sciences, 30 Pembroke Court, Chatham Maritime, Kent, ME4 4UF, United Kingdom

(c) Tissue Engineering and Regenerative Medicine 2014

Erratum to: Tissue Engineering and Regenerative Medicine (2014), 11(5): pp 405-413

The author, Jun Su Park, should be corrected to Jun DOI 10.1007/s13770-014-0041-8

Soo Park. The correct name is also shown here.

The original version of this article contained an error in one of the authors' first name.

The online version of the original article can be found under DOI: 10.1007/s13770-014-0041-8

\section{${ }^{*}$ Corresponding author}

e-mail: peter@catholic.ac.kr (Seok Jung Kim)

Department of Orthopedic Surgery, College of Medicine,

The Catholic University of Korea 